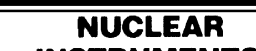

\title{
Short induction gap gas electron multiplier (GEM) for X-ray spectroscopy
}

\author{
J.A. Mir ${ }^{\text {a,* }}$, R. Stephenson ${ }^{\text {a }}$, N.J. Rhodes ${ }^{\text {a }}$, E.M. Schooneveld ${ }^{\text {a }}$, \\ J.F.C.A. Veloso ${ }^{\mathrm{b}, \mathrm{c}}$, J.M.F. Dos Santos \\ ${ }^{a}$ CCLRC Rutherford Appleton Laboratory, Chilton, Didcot, Oxon OX11 0QX, UK \\ ${ }^{\mathrm{b}}$ Departmento de Fisica, Universidade de Coimbra, P 3004516 Coimbra, Portugal \\ ${ }^{\mathrm{c}}$ Departmento de Fisica, Universidade de Aveiro, P 3810193 Aveiro, Portugal
}

Available online 20 November 2006

\begin{abstract}
Experimental work was carried out to evaluate the performance of a Gas Electron Multiplier (GEM) operated with a micromesh readout plane that enabled the induction gap to be set at $50 \mu \mathrm{m}$. We measured the essential operational parameters of this system using $\operatorname{Ar}(75 \%)$-isobutane $(25 \%)$ as the counter gas mixture. The measurements included the effective gain, effective gain stability, and the X-ray energy resolution using a $5.89 \mathrm{keV} \mathrm{X-ray} \mathrm{source.} \mathrm{These} \mathrm{studies} \mathrm{demonstrated} \mathrm{several} \mathrm{advantages} \mathrm{of} \mathrm{the} \mathrm{current} \mathrm{system} \mathrm{when}$ compared with the standard operation, such as lower operational voltages, higher effective gains and improved effective gain stability. (C) 2006 Elsevier B.V. All rights reserved.
\end{abstract}

PACS: 29.40.Cs; 29.40.Gx; 85.60.Gz

Keywords: Gas Electron Multiplier; GEM; Effective gain; X-ray energy resolution

\section{Introduction}

A typical Gas Electron Multiplier (GEM) configuration [1] sets the induction gap at $1 \mathrm{~mm}$ or more where the induction gap is defined as the distance between the bottom GEM electrode and the readout plane. It is well known that Kapton-based GEMs are susceptible to absorption by water vapour and other counter gases [2]. One of the consequences of the absorption process is the sagging of the GEM foil thereby changing the induction gap from its initial set value. However, the effective gain of a GEM is strongly dependant upon the induction field $[3,4]$. As a result, GEM sagging leads to an effective gain instability [4]. One way to circumvent this is to introduce dielectric pillars between the GEM foil and the readout plane at regular interval [5]. In the present study, we have used a standard GEM that was coupled with a micromesh [6] readout plane with $50 \mu \mathrm{m}$ tall Kapton pillars patterned on the micromesh at $2 \mathrm{~mm}$ pitch. The $50 \mu \mathrm{m}$ induction gap had

\footnotetext{
*Corresponding author. Tel.: + 44123544 6262; fax: + 441235446863.

E-mail address: J.A.Mir@rl.ac.uk (J.A. Mir).
}

several distinct operational advantages. For example, the effective gain stability was improved owing to a good induction gap definition and the absolute voltage needed to sustain a particular induction field was lowered by a factor of 20 .

\section{Method}

The X-ray sensitive area of the present detection system consisted of a $10 \mathrm{~mm} \times 10 \mathrm{~mm}$ GEM, a micromesh and a $100 \mu \mathrm{m}$ thick aluminium foil that defined the drift window. The drift distance was set at $5 \mathrm{~mm}$ whereas the induction gap was set at $50 \mu \mathrm{m}$. The GEM used here was of a standard geometry and was fabricated at the CERN TS-DEM workshop and consisted of a $50 \mu \mathrm{m}$ thick copper clad $(5 \mu \mathrm{m})$ Kapton foil with $70(50) \mu \mathrm{m}$ holes patterned at $140 \mu \mathrm{m}$ hole pitch. The micromesh was also manufactured at CERN TS-DEM workshop and consisted of a $5 \mu \mathrm{m}$ thick Copper mesh with $25 \mu \mathrm{m}$ holes etched at a pitch of $50 \mu \mathrm{m}$ with $50 \mu \mathrm{m}$ tall and $150 \mu \mathrm{m}$ diameter Kapton pillars distributed at $2 \mathrm{~mm}$ intervals [6]. 
In the experimental studies described in the following sections, a Mn-K X-ray $(5.89 \mathrm{keV})$ beam illuminated the detector drift space perpendicular to the GEM and micromesh planes through an entrance window near the end wall of the detector chamber. The entrance window consisted of $100 \mu \mathrm{m}$ mylar mesh with $100 \mathrm{~nm}$ aluminium coating on one side. The entrance window was located approximately $10 \mathrm{~mm}$ away from the drift window. In this study, the detection chamber was operated at a constant gas flow rate where the gas composition was controlled by Brooks mass-flow controllers (model 5850E) built into a rig that was constructed using stainless-steel tubing. An argon $(75 \%)$ and isobutane $(25 \%)$ counter gas mixture was used throughout this study. The ambient pressure and the temperature of the detector box were recorded with every measurement. The global count rates used throughout these studies were kept in the region of $10 \mathrm{kHz}$.

The drift electrode and the GEM mesh were operated negative with respect to the micromesh that was held close to earth. The micromesh was connected electrically to an Ortec preamplifier (model 142A). The preamplifier output was then fed into an Oretc shaping amplifier (model 575A) with shaping time constants adjusted to $0.5 \mu$ s. The bipolar output of the shaping amplifier was in turn fed into an Ortec pulse height analyser (Ortec Trump-PCI-2 K plug in card with Maestro-32 software for Windows). The effective gain and the $\mathrm{X}$-ray energy resolution were examined as a function of the induction field, $E_{\mathrm{I}}$, and the voltage differences applied across the GEM holes, $\Delta V_{\mathrm{GEM}}$. The drift field, $E_{\mathrm{d}}$, was maintained at approximately $3.5 \mathrm{kV} / \mathrm{cm}$ throughout these studies. The effective gain stability was also studied by monitoring the detector gain for a period of one month.

\section{Effective gain and $X$-ray energy resolution}

Fig. 1 shows the variation of the effective gain as a function of the induction field for the range $E_{\mathrm{I}}=0.4-40 \mathrm{kV} / \mathrm{cm}$ for a number of different voltages across the GEM holes $\left(\Delta V_{\mathrm{GEM}}=400,450\right.$ and $\left.500 \mathrm{~V}\right)$. The effective gain increases almost linearly with increasing induction field. When $E_{\mathrm{I}}$ exceeds values above $15 \mathrm{kV} / \mathrm{cm}$, further electron multiplication begins in the induction region (parallel plate amplification mode).

Fig. 2 shows the variation of the X-ray energy resolution at $5.89 \mathrm{keV}$ as a function of the induction field for different voltages across the GEM holes. For induction fields, $E_{\mathrm{I}}$, lower than $2 \mathrm{kV} / \mathrm{cm}$, the X-ray energy resolution was rather poor due to most electrons being directed to the lower GEM electrode. The optimum X-ray energy resolution was observed in the induction field region $2-15 \mathrm{kV} / \mathrm{cm}$, beyond which rapid deterioration in the $\mathrm{X}$-ray energy resolutions occurred.

Fig. 3 shows the variation of the effective gain as a function of voltage applied across the GEM holes for a number of different induction fields $\left(E_{\mathrm{I}}=6,12,20,30\right.$ and

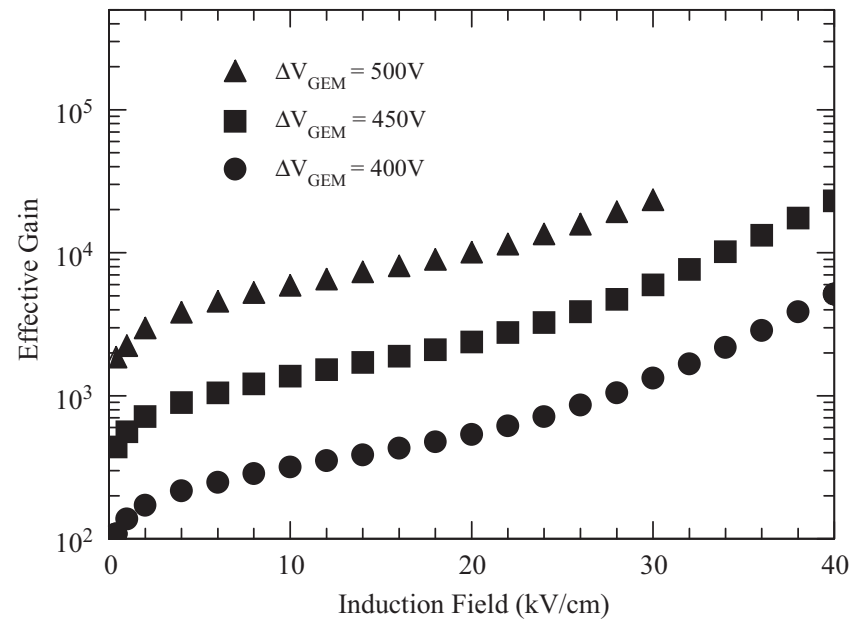

Fig. 1. A plot of the effective gain as a function of the induction field using $\operatorname{Ar}(75 \%)$-isobutane $(25 \%)$ for $\Delta V_{\mathrm{GEM}}=400,450$ and $500 \mathrm{~V}$. In all cases the drift field $E_{\mathrm{d}}=-3.5 \mathrm{kV} / \mathrm{cm}$.

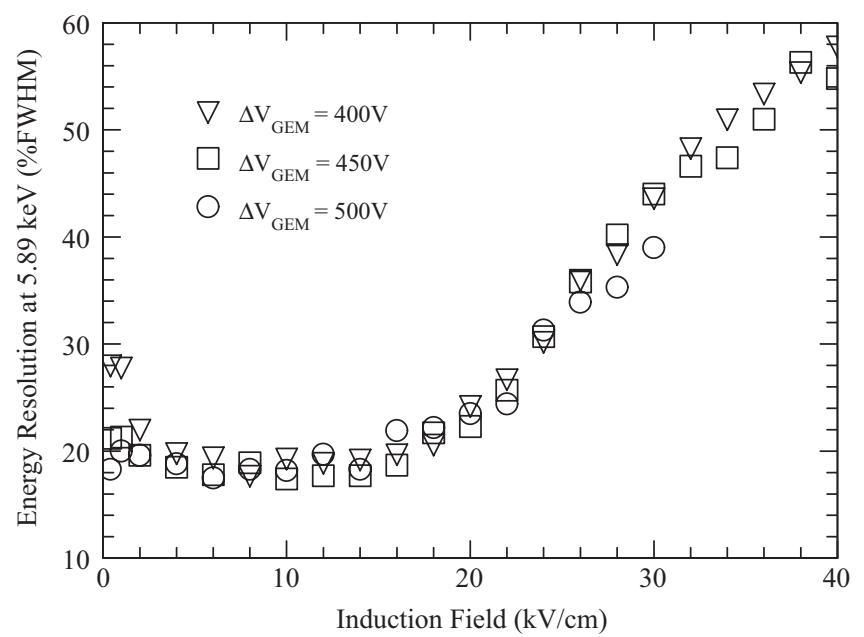

Fig. 2. X-ray energy resolution (\% FWHM) of the Mn K X-rays $(5.89 \mathrm{keV})$ as a function of the induction field using $\mathrm{Ar}(75 \%)$-isobutane $(25 \%)$ for $\Delta V_{\mathrm{GEM}}=400,450$ and $500 \mathrm{~V}$. In all cases $E_{\mathrm{d}}=-3.5 \mathrm{kV} / \mathrm{cm}$.

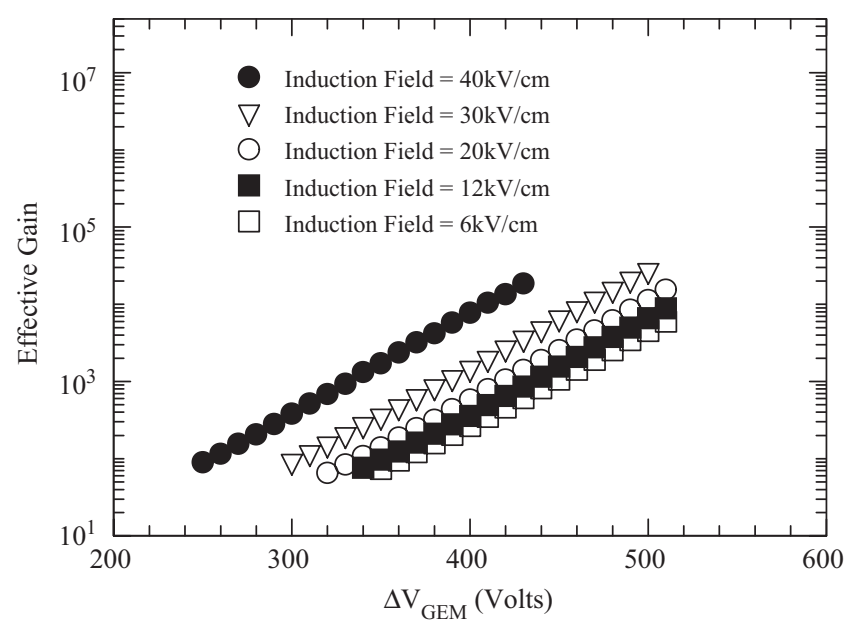

Fig. 3. Effective gain as a function of the potential across the GEM holes $\left(\Delta V_{\text {GEM }}\right)$ using $\operatorname{Ar}(75 \%)$-isobutane $(25 \%)$ for a number of different induction fields. In all cases the drift field $E_{\mathrm{d}}=-3.5 \mathrm{kV} / \mathrm{cm}$. 
$40 \mathrm{kV} / \mathrm{cm}$ ). The highest effective gain at the lowest induction field used here was found to be approximately 6000. Increasing the induction fields to values higher than $6 \mathrm{kV} / \mathrm{cm}$ shifted the effective gain-voltage $\left(\Delta V_{\mathrm{GEM}}\right)$ characteristics. The highest gain observed was approximately 27,000 when the induction field was set at $30 \mathrm{kV} / \mathrm{cm}$.

The X-ray energy resolution at $5.89 \mathrm{keV}$ associated with each curve in Fig. 3 is plotted in Fig. 4. It is clear from Figs. 1-4 that although effective gain increased with induction fields higher than $6 \mathrm{kV} / \mathrm{cm}$, this was accompanied by a degradation in the corresponding X-ray energy resolution [5]. Fig. 5 shows an optimum pulse height spectrum at $5.89 \mathrm{keV}$ when the GEM was operated at an effective gain of $1900\left(\Delta V_{\mathrm{GEM}}=470 \mathrm{~V}, E_{\mathrm{I}}=6 \mathrm{kV} / \mathrm{cm}\right.$, $\left.E_{\mathrm{d}}=-3.5 \mathrm{kV} / \mathrm{cm}\right)$. The X-ray energy resolution derived from this figure was found to be $15.2 \%$ FWHM.

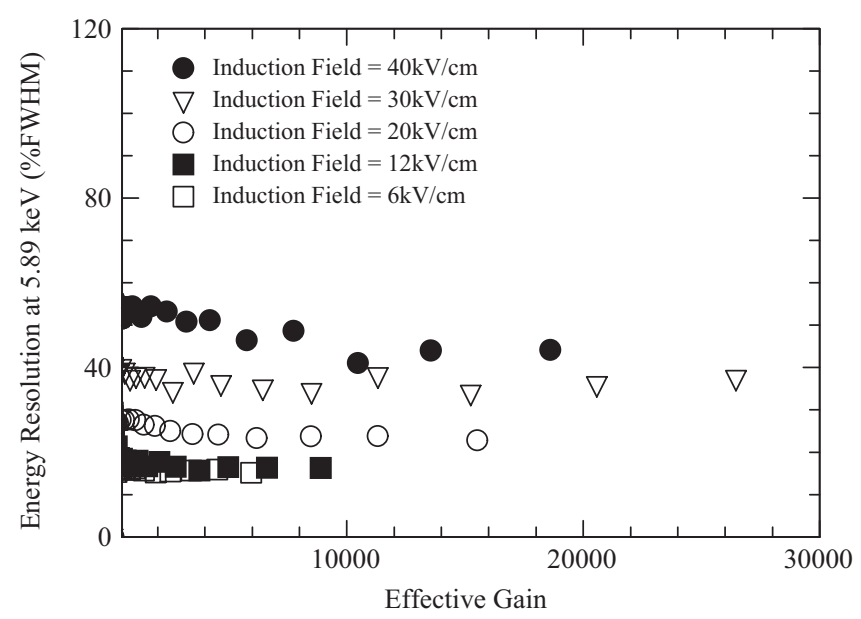

Fig. 4. X-ray energy resolution (\%FWHM) of the Mn K X-rays $(5.89 \mathrm{keV})$ as a function of the effective gain using $\mathrm{Ar}(75 \%)$-isobutane $(25 \%)$ for a number of different induction fields. In all cases the drift field $E_{\mathrm{d}}=-3.5 \mathrm{kV} / \mathrm{cm}$.

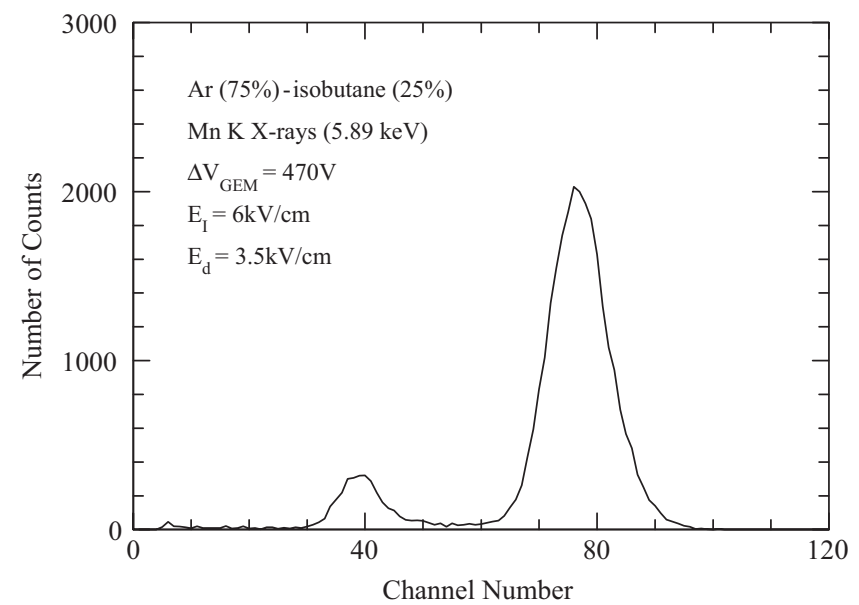

Fig. 5. A typical pulse height spectrum for $5.89 \mathrm{keV}$ X-rays using the GEM at an effective gain of 1900 in $\operatorname{Ar}(75 \%)$-isobutane $(25 \%)$ with $\Delta V_{\mathrm{GEM}}=470 \mathrm{~V}, E_{\mathrm{I}}=6 \mathrm{kV} / \mathrm{cm}$ and $E_{\mathrm{d}}=-3.5 \mathrm{kV} / \mathrm{cm}$.

\section{Effective gain stability}

The effective gain of flow type gaseous detectors is influenced by the ratio $P / T$ [7 and references therein], where $P$ is the gas pressure in $\mathrm{mB}$ and $T$ is its absolute temperature in K. Fig. 6 shows the effective gain of the present GEM over a one month period plotted as a function of $P / T$. Fitting an exponential curve to the data gives the effective gain sensitivity of the detector to changes in the ambient conditions. In the present case, this evaluates to $1.55 \mathrm{~K} / \mathrm{mB}$ as shown in Fig. 6. Assuming that $P$ is roughly constant at around $1000 \mathrm{mB}$ and allowing for maximum temperature excursion of $\pm 5^{\circ} \mathrm{C}$ from $20{ }^{\circ} \mathrm{C}$ would result in a maximum gain excursion of $\pm 8.9 \%$.

\section{Conclusion}

A short gap GEM was successfully operated with an induction gap of $50 \mu \mathrm{m}$ to investigate parameters relevant to the X-ray detection. These included measurements of the effective gain and X-ray energy resolution as a function of the induction field and the voltage applied across the GEM holes.

The highest effective gain was found to be approximately 27000 when the voltage across the GEM holes was set at $500 \mathrm{~V}$ and induction field at $30 \mathrm{kV} / \mathrm{cm}$. However, the best $\mathrm{X}$-ray energy resolution was found to be $15.2 \%$ FWHM when the voltage across the GEM holes was $470 \mathrm{~V}$ (effective gain 1900) and the induction field was $6 \mathrm{kV} / \mathrm{cm}$.

The effective gain shift with time experienced during our previous studies [4] was overcome by the simple introduction of $50 \mu \mathrm{m}$ tall Kapton pillars between the lower GEM electrode and the readout plane. Effective gain shifts observed during the one month run were consistent with the changes in ambient pressure and temperature over that period.

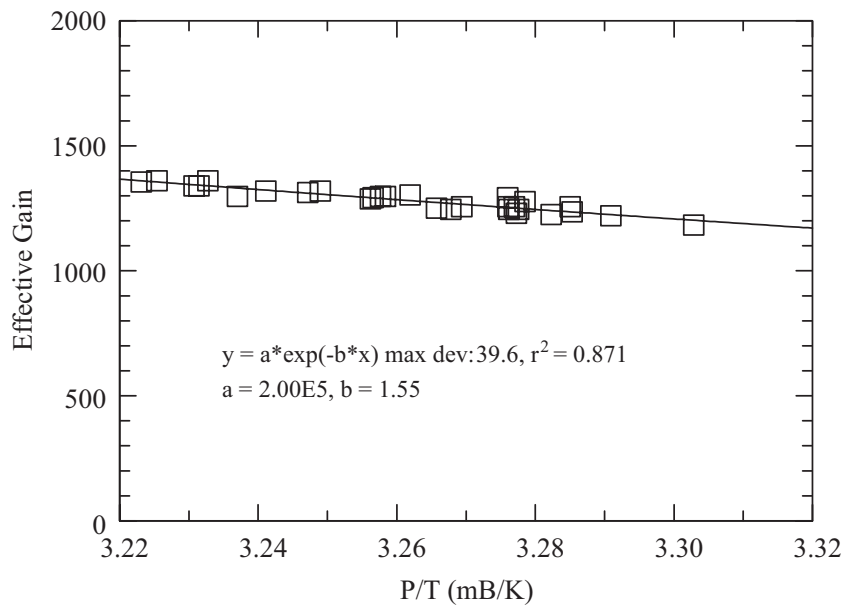

Fig. 6. The effective gain measured over a period of one month using Ar (75\%)-isobutane $(25 \%)$ as a function of the ambient parameter $P / T$. $\Delta V_{\mathrm{GEM}}=450 \mathrm{~V}, E_{\mathrm{I}}=6 \mathrm{kV} / \mathrm{cm}$ and $E_{\mathrm{d}}=-3.5 \mathrm{kV} / \mathrm{cm}$. 


\section{References}

[1] F. Sauli, et al., GEM: a new concept for electron amplification in gas detectors, Nucl. Instr. and Meth. A 386 (1997) 531.

[2] J.I. Collar, et al., Nucl. Instr. and Meth. A 515 (2003) 439.

[3] F. Sauli, et al., Further developments and beam tests of the gas electron multiplier (GEM), Nucl. Instr. and Meth. A 419 (1998) 410 .
[4] J.A. Mir, et al., Operational characteristics of a GEM-MSGC system for X-ray detection, IEEE Trans. Nucl. Sci. NS-52 (6, Part 2) (2005) 2927.

[5] G. De Lentdecker, et al., Nucl. Instr. and Meth. A 459 (2001) 211.

[6] Y. Giomataris, Nucl. Instr. and Meth. A 419 (1998) 239.

[7] J.E. Bateman, et al., Studies of the gain properties of microstrip gas counters relevant to their application as X-ray and electron detectors, IEEE Trans. Nucl. Sci. NS-49 (4, Part 1) (2002) 1644. 